\title{
Vibration analysis of airfoil on hereditary deformable suspensions
}

\author{
Botir Usmonov and Quvvatali Rakhimov* \\ Tashkent University of Information Technologies, Amir Temur street, 108, Tashkent, 100200, \\ Uzbekistan
}

\begin{abstract}
This paper describes the analyses of the nonlinear vibrations and dynamic stability of an airfoil on hereditary-deformable suspensions. The model is based on two-degree-of-freedom structure in geometrically nonlinear statements. It provides justification for the choice of the weakly singular Abelian type kernel, with rheological parameters. To solve problems of viscoelastic system with weakly singular kernels of relaxation, a numerical method has been used, based on quadrature formulae. With a combination of the Galerkin and the presented method, problems of nonlinear vibrations and dynamic stability in viscoelastic two-degree-offreedom structure have been solved. A comparison of the results obtained via this method is also presented. In all problems, the convergence of the Galerkin method has been investigated. The implications of material viscoelasticity on vibration and dynamic stability are presented graphically.
\end{abstract}

\section{Introduction}

At recent years, the mechanics of composite materials has great impact to the development of the aerospace industry. There are many problems such as deformation, durability, vibrations, and dynamic stability of the structures made from composite materials. The study of these problems in context of composite materials helps to solve many problems in the aerospace industry such as weight, strength, and reliability in the design and engineering. Interest in problems of deformation, durability, vibrations, and dynamic stability of structures made of composite material is prompted by the fact that they are the main load-bearing elements in, etc. Strong demand for reliable elements of the structure made from advanced composite materials in the engineering requires better mathematical and mechanical models of viscoelastic systems. Therefore, the development of efficient algorithms for viscoelastic system to solve nonlinear problems of vibration and dynamic stability is become one of priorities of the mechanics of composite materials. Many composite material constants are distinct viscoelastic properties [1], [2], [3], [4], [5]. Viscoelastic systems made of composite materials having different structure have been described previously [8], [15].

\footnotetext{
* Corresponding author: quvvatali.rahimov@gmail.com
} 
Various studies have been devoted to the solution of elastic structural (isotropic cases and orthotropic cases) problems [1], [2], [3], [4]. Even if the problems were solved in the viscoelastic formulation, in many cases the viscoelastic characteristics of the material were only taken into account in a restricted context [6], [7], and [8]. Actually, mathematical models of problems of viscoelastic systems based on these assumptions cannot describe real processes in constructions and the influence of dynamic loadings [3], [4]. The lack of assumptions leads to incorrect approximation of relaxation and creep processes in the initial stage of deformation. The choice of assumptions should not be casually undertaken. In cases where a similar problem is solved in a linear statement, the decision is reduced to the application of various integrated transformations, e.g. Laplace, Laplace-Carson, etc.[7], [9], [10] and [8]. If they are solved in a nonlinear statement, it can be obtained as the solution of a system of integro-differential equations, where, by way of differentiation, it may be reduced to the solution of ordinary differential equations, which in most cases are solved by the method of Runge-Kutta or finite element method [5], [6], [7], and [10]. At present, the existing methods do not allow the resolution of problems involving weakly singular kernels of the Koltunov, Rzhanitsyn, Abel and Rabotnov types, among others [2] and [3].

According to the numerical method [12], [13], [14] and [15] developed by F. Badalov on the basis of quadrature rules, it is now possible to solve the system of nonlinear integrodifferential equations with weakly singular kernels of the Abel and other types. This method provides results of a reasonably high accuracy and is universal. It enables the resolution of a wide class of dynamic problems of the theory of viscoelasticity and is economical from the point of view of calculation time [13], [14], [15].

The purpose of this work is the study of nonlinear vibrations and the dynamic stability of the airfoil with viscoelastic suspensions. At present time, the existing methods do not allow solve of problems involving weakly singular kernels including [2] and [3].

According to the numerical method [14] and [15] based on quadrature formulas; it is possible to solve the system of nonlinear integro-differential equations with weakly singular kernels. This method provides a high accuracy of results.

\section{Structural model}

The constitutive relation between stress and strain on suspensions of the airfoil is assumed to have the form

$$
\sigma=\left(1+R^{*}\right)\left(E \varepsilon+E_{1} \varepsilon^{3}\right)
$$

and the airfoil structure has cubic nonlinearity from [11-19] that is, $\tau=\left(1+R^{*}\right)\left(G \gamma+G_{1} \gamma^{3}\right), \gamma=-z \theta_{x}$, where $E_{1}$ and $G_{1}$ are physical nonlinearity coefficients, which are less than zero $\left(E_{1}<0, G_{1}<0\right)$ for soft materials and greater than zero $\left(E_{1}>0, G_{1}>0\right)$ for rigid materials.

Assume that a rheological property of an airfoil structure is governed under cubic nonlinearity law of the viscoelasticity [15]. Then according to the variation principle of hereditary theory of viscoelasticity [2,3] kinetic and potential energy describes by next expressions:

$$
\begin{gathered}
K=\frac{1}{2}\left(c_{11} q^{2}+c_{22} \dot{r}^{2}+2 c_{12} \dot{q} \dot{r}\right) \\
P=\frac{1}{2}\left\{a_{1} q\left[\left(q-0.5 \gamma q^{3}\right)-2 R^{*}\left(q-\gamma q^{3}\right)\right]+a_{2} r\left[\left(r-0.5 \gamma r^{3}\right)-2 R^{*}\left(r-\gamma r^{3}\right)\right]\right\}
\end{gathered}
$$

where $\gamma, \gamma_{1}$ are parameters of nonlinearity, $c_{i j}$ is coefficient of inertia and $a_{i j}$ is coefficient of rigidity and $c_{11}=m, c_{12}=c_{21}=-m b, c_{22}=m\left(r^{2}+b^{2}\right)$. 
Substituting equations (2) and (3) into Lagrange equation $\frac{\partial L}{\partial U_{i}}-\frac{d}{d t} \frac{\partial L}{\partial U_{i}}=0$, where $L=P-T, U_{1}=q(t), U_{2}=r(t)$ yields

$$
M \ddot{U}+K\left(1-R^{*}\right)\left(U-\gamma U^{3}\right)=0 .
$$

The initial conditions are

$$
U(0)=U_{0}, \dot{U}(0)=\dot{U}_{0}
$$

The equation (4) is nonlinear integro-differential equation of motion of airfoil structure, where $b$ is coupling term for bending-torsion vibration of airfoil model. When axis of rigidity and axis of the centre of gravity are in one line, $b=0$ and system (4) is separated into two independent equations. First equation is describes vertical vibrations, and second equation is describes torsional motion of an airfoil model of wing. Because such constructive placement of axis is difficult, vibration of the system is considered as coupled bending-torsion vibration of an airfoil model.

In the equation (4) other terms describes in following matrixes $U=\left(\begin{array}{l}q(t) \\ r(t)\end{array}\right)$ is matrix of generalized displacements, $M=\left(\begin{array}{cc}c_{11} & c_{12} \\ c_{21} & c_{22}\end{array}\right)$ is mass matrix, and $K=\left(\begin{array}{cc}a_{11} & 0 \\ 0 & a_{22}\end{array}\right)$ is rigidity matrix.

System of nonlinear weak singular equations (4) with initial conditions (5) describes mathematical model of the classical vibration problem. System of equation (4) is general, which can be divided into 2 particular vibration problems. Then one can have two degree of freedom vibration problem, mentioned above. When $R^{*}=0, \gamma=0$, system of equations is linear problem of vibration of the elastic airfoil model of wing [1,2], and at $R^{*}=0$ will have problem of the vibration of airfoil model of wing from nonlinear elastic material. In the linear case, when $\gamma=\gamma_{1}=0$ exact solution of the equations (4) at initial conditions (5) is obtained by considering: $c_{11}=c_{1}, c_{12}=c_{21}=0$, and $c_{22}=c_{2}$.

In the nonlinear case, the task is become more complex and required to apply numerical methods. Therefore, in the equations (4) are setting dimensionless terms, such as $\theta_{1}=1+\left(\frac{b}{r}\right)^{2}, \theta_{2}=\chi\left(\frac{b}{r}\right)^{2}, b h=u, \alpha=w, \bar{t}=\sqrt{\frac{c_{1}}{m}} t, \chi=\frac{c_{2}}{c_{1} b^{2}}$.

After introducing above described terms into equation (4), it is become

$$
\begin{aligned}
& \ddot{W}+\theta_{1}\left(1-R^{*}\right)\left[\left(W-\gamma W^{3}\right)+\frac{\theta_{2}}{\theta_{1}}\left(U-\gamma_{2} U^{3}\right)\right]=0 \\
& \ddot{U}+\left(\frac{b}{r}\right)^{2}\left(1-R^{*}\right)\left[\left(W-\gamma W^{3}\right)+\frac{\theta_{2}}{\theta_{1}}\left(U-\gamma_{2} U^{3}\right)\right]=0
\end{aligned}
$$

Exact solution of system of the weak singular integro-differential equations are has mathematical difficulties. For a complete solution of integro-differential equations (IDE) (6), it is necessary to add boundary and initial conditions. Initial conditions given by the following expressions:

$$
\left.W\right|_{t=0}=W_{0},\left.\quad \dot{W}\right|_{t=0}=0,\left.U\right|_{t=0}=U_{0},\left.\dot{U}\right|_{t=0}=0 .
$$

Therefore, for solution of equations (6) is used a method of elimination of weak singularities of integro-differential equations developed by Badalov [15]. 


\section{Discrete model}

The exact solution of system weak-singular IDE (6) in partial derivative represents significant mathematical difficulties. The approximate solution of equation (6), according to the method of elimination of weak-singularity features of a integral and IDE [15], is based on the linear recurrent system of the algebraic equations:

$$
\begin{gathered}
W_{n}=W_{0}-\sum_{j=0}^{n-1} C_{j}\left(t_{n}-t_{j}\right)\left\{\theta_{1}\left(W_{j}-\gamma W_{j}^{3}\right)+\theta_{2}\left(U_{j}-\gamma U_{j}^{3}\right)-\frac{\varepsilon}{a} \sum_{k=0}^{j} B_{k} e^{-\beta t_{k}} D_{1}\right\} \\
U_{n}=U_{0}-\left(\frac{b}{r}\right)^{2} \sum_{j=0}^{n-1} C_{j}\left(t_{n}-t_{j}\right)\left\{\theta_{1}\left(W_{j}-\gamma W_{j}^{3}\right)+\chi\left(U_{j}-\gamma U_{j}^{3}\right)-\frac{\varepsilon}{a} \sum_{k=0}^{j} B_{k} e^{-\beta t_{k}} D_{2}\right\}
\end{gathered}
$$

where

$$
D_{1}=\left(\theta_{1}\left(W_{j-k}-\gamma W_{j-k}^{3}\right)+\theta_{2}\left(U_{j-k}-\gamma U_{j-k}^{3}\right)\right), \quad D_{2}=\left(\left(W_{j-k}-\gamma W_{j-k}^{3}\right)+\chi\left(U_{j-k}-\gamma U_{j-k}^{3}\right)\right),
$$

$$
t_{n}=n \Delta t, W\left(t_{n}\right)=W_{n}, U\left(t_{n}\right)=U_{n}, C_{j}=\Delta t, \quad j=\overline{1, n-1}, C_{0}=C_{n}=\frac{\Delta t}{2}, B_{k}=\frac{\Delta t^{a}}{2}\left[(k+1)^{a}-(k-1)^{a}\right],
$$

$k=1, j-1, B_{0}=\frac{\Delta t^{a}}{2}, B_{j}=\frac{\Delta t^{a}}{2}\left[j^{a}-(j-1)^{a}\right]$.

Solution of system linear recurrent algebraic equations (8) allows obtain numerical values of functions $W, U$ for $i=\overline{1, N} ; n=1,2, \ldots$.

\section{Numerical example}

In this section is represented a numerical validation of the problem in Figure 1 by the integration method. The solution of the excitation of the problem is described by equation (7). This equation is used for numerical calculation of a dynamic responses of the viscoelastic suspensions of the airfoil. The dynamic response of the system is investigating under free vibration. In detail, the effects of the nonlinearity and viscosity parameters of the material are investigated, and analyzed after the numerical implementation of the equation (7). For the small influence, the relaxation $\beta$ is fixed in all experiments as constant based on the previous works. In the experiments were explored the influence the singularity and viscosity parameters. Nonlinearities are imposed by giving the nonlinearity parameter.

\subsection{Free vibration of the airfoil with viscoelastic suspensions}

Before investigating the vibratory behavior is formulated an initial conditions and suspension material constants $\chi=0.9 ;(r / b)^{2}=0.44 ; \varepsilon=0.0 ; \alpha=0.0 ; \beta=0.0 ; \Delta t=0.1$

For realistic study Goland's cantilever wing model was selected to simulate the dynamic response and stability analysis. It has following parameters:

- Wing half span $l=20 \mathrm{ft}$

- Wing chord $c=6 \mathrm{ft}$

- Mass $m=0.746$ slugs per $\mathrm{ft}$

- $\quad$ Radius of gyration $=25 \%$ of chord

- $\quad$ Span wise elastic axis $=33 \%$ of chord

- Center of gravity $=43 \%$ of chord

- Bending rigidity $E I=23.65 \times 10^{6} \mathrm{lb} \cdot \mathrm{ft}^{2}$

- Torsion rigidity $G J=2.39 \times 10^{6} \mathrm{lb} \mathrm{ft}^{2}$ 
Main aim is to explore the influence of material properties to the free vibrated motion of the airfoil. Therefore, the first analyses and compares perfect elastic linear and nonlinear $(\gamma=-0.2)$ (Figure 1) with linear and nonlinear $(\gamma=-0.2)$ viscoelastic motion of the suspensions (Figure 2). When the viscosity parameter is varied by $0,0.1$, and 0.2 , the vibratory behaviors of the linear and nonlinear cases are depicted by Figures 1 and 2 . Calculation is provided for both cases, for the elastic and viscoelastic models with different structural nonlinearity parameters.

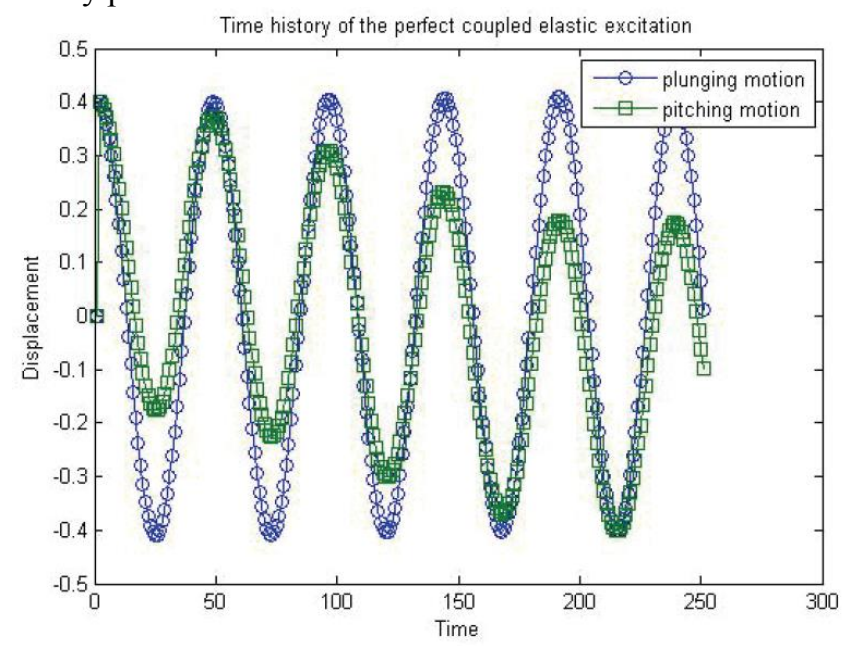

Fig.1. Time history of the perfect elastic wing model - $\varepsilon=0$

If in Figures 1 and 2 was analysed the free vibration at perfect elastic conditions with out any affects of structural conditions. This graph demonstrated perfect pitching and plunging displacements for $\chi=0.1 ;(r / b)^{2}=0.44 ; \varepsilon=0.0 ; \alpha=0.0 ; \beta=0.0 ; \Delta t=0.1$. As analogous time history of the perfect elastic pitching and plunging displacements for Goland wing model with linear and nonlinear viscoelastic suspensions are demonstrated in Fig.4 and Fig.5, which illustrate different rate of amplitude and frequency for plunging and pitching compare to the Fig. 1-2. Coupling motion has influence into frequency of pitching motion, which is observed in Fig. 5.

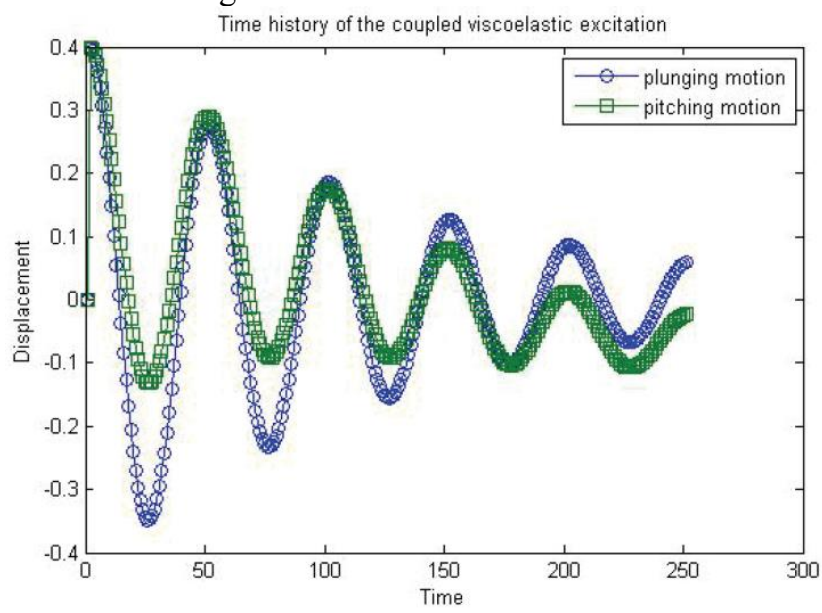

Fig. 2. Time history of the perfect elastic wing model $-\varepsilon=0.2$ 


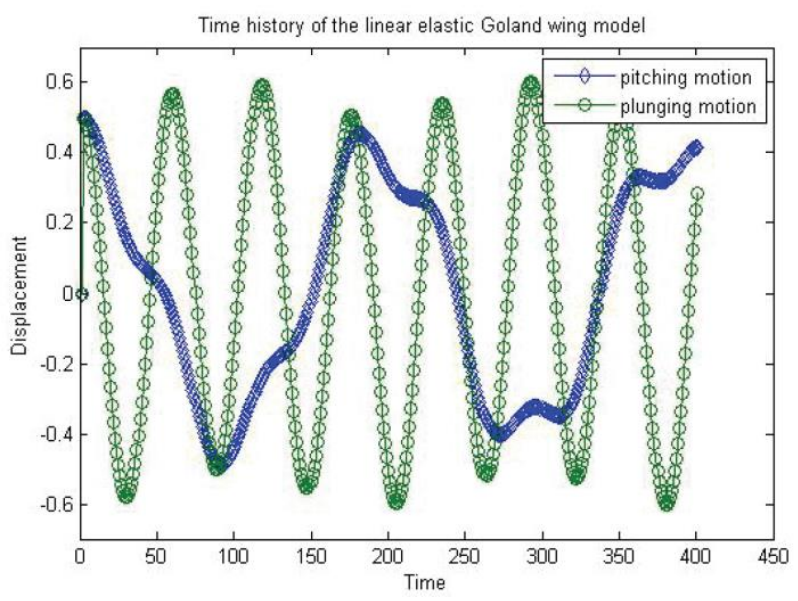

Fig. 3. Time history of the linear elastic Goland wing model- $\varepsilon=0$

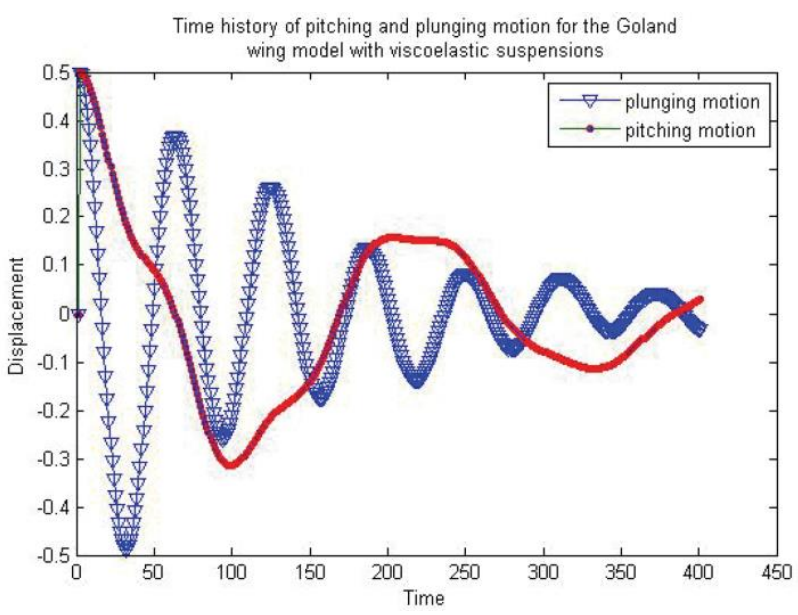

Fig. 4. Time history of the linear elastic Goland wing model- $\varepsilon=0.2$

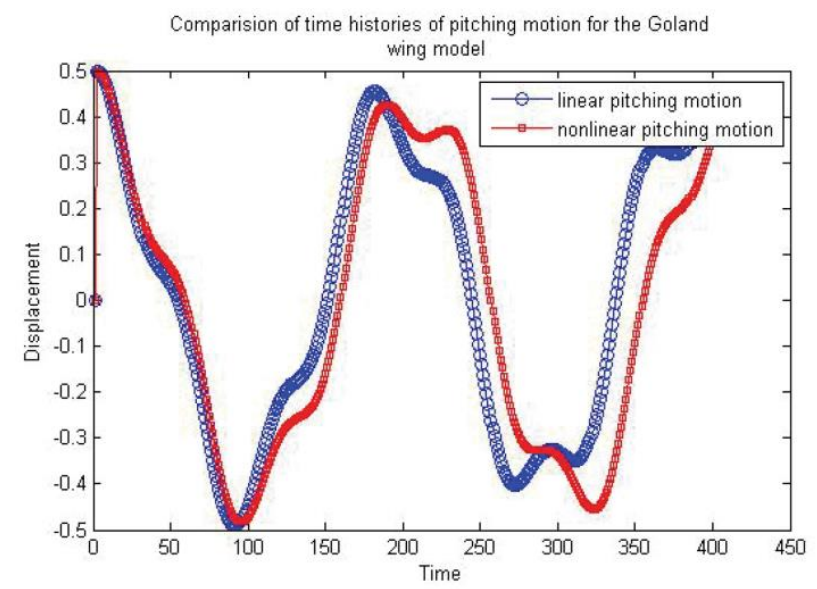

Fig.5. Comparison of the pitching motions for the elastic suspension 
The results show (see Figures 1-2) that viscosity parameter $\varepsilon$ affects to the amplitude and excitation period. With the given parameter, the vibratory curve of the shows stiffening effect in Figures 1 and 3. However, as the viscosity parameter (see Figure 2 and Figure 4) increases, the displacement in both cases goes down faster. In other words, the free vibration with much viscosity parameter is damping the vibratory behavior faster.
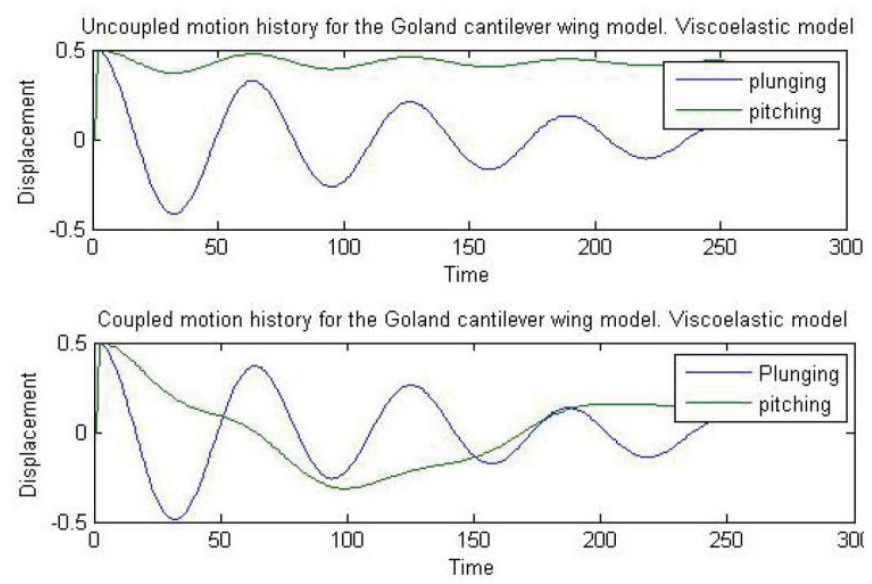

Fig. 6. Uncoupled and Coupling motion history for Goland wing model

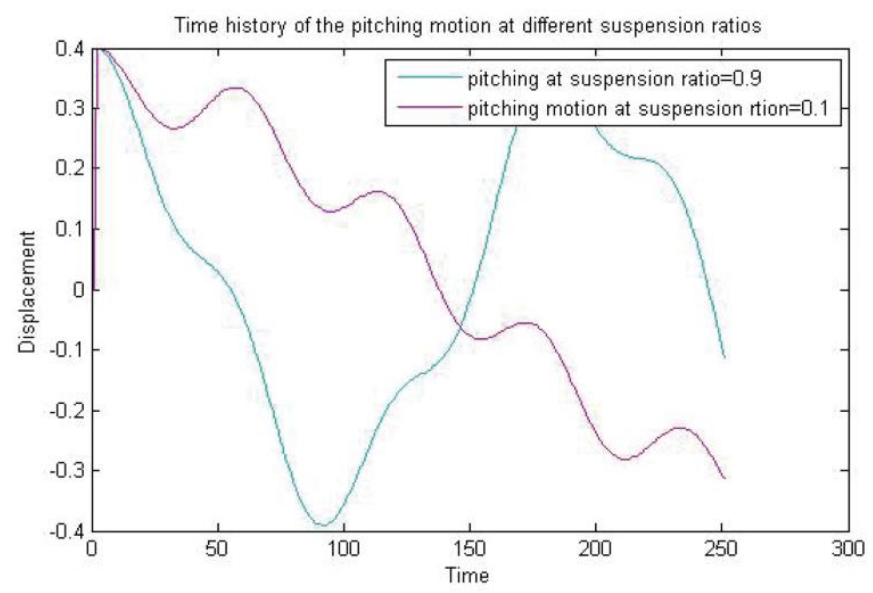

Fig. 7. Time history of the pitching motion at different suspension ratios

\section{Conclusion}

Application of the variation method to viscoelastic problems is demonstrated in this work as an incremental integration in time.

The constitutive relation (stress-strain) was used in a form of a hereditary law with relaxation kernel represented by an Abelian type weak- singularity.

The general procedure of solution of the nonlinear integro-differential equations for an airfoil with hereditary-deformable suspensions is formulated and analyzed. The analysis is given for the general vibratory problem of the airfoil. 


\section{References}

1. A. Ilyushin and B. Pobadrya, Fundamentals of the mathematical theory thermoviscoelasticity (Science, Moscow, 1970)

2. Y. Rabotnov, Elements of the Hereditary Mechanics of Solids (Nauka, Moscow, 1977)

3. M. Koltunov, Creep and Relaxation (Viszhaya Shkola, Moscow, 1976)

4. R. Christensen, Theory of Viscoelasticity (Academic Press, New York, 1971) https://doi.org/10.1016/B978-0-12-174252-2.X5001-7

5. A. Malmeyster, V. Tamuzh, and G. Teters, Resistance of Composite Materials (Zinatne, Riga, 1980)

6. A. Bogdanovich, Nonlinear Dynamic Problems for Composite Cylindrical Shells (Elsevier Science Publishers Ltd, New York, 1993) https://doi.org/10.1201/9781482296617

7. C. W. Bert, Material damping: An introductory review of mathematic measures and experimental technique, Journal of Sound and Vibration, 29, 129-153 (1973) https://doi.org/10.1016/S0022-460X(73)80131-2

8. S. Menon and J. Tang, A State-space approach for the dynamic analysis of viscoelastic systems, Computers and structures, 82, 1123-1130 (2004)

9. A. Muravyov, Analytical solutions in the time domain for vibration problems of discrete viscoelastic systems, Journal of Sound and Vibration, 199, 337-348 (1997)

10. D. Golla and P. Hughes, Dynamics of viscoelastic structures - a time domain, finite element formulation, Journal of Applied Mechanics, 52, 897-906 (1985)

11. F. Badalov and B.Usmonov, Vibrations hereditarity-deformable of a airfoil with aileron in an air flow, Academy of Science of Uzbekistan, Tashkent, (2004), pp. 51-57.

12. F. Badalov, T.Kholmatov, and G. Shodmonov, About one generalization of Newmark's method for solving IDE of dynamic problems of viscoelastic linear theory, Dokl. Akad. Nauk Resp. Uzbekistan, 8 (1999)

13. F. Badalov, K. Eshmatov, and M. Yusupov, On certain methods of solving systems of integro-differential equations encountered in viscoelasticity problems, Journal of Applied Mathematics and Mechanics, 51, 867-871 (1987) https://doi.org/10.1016/0021$\underline{\text { 8928(87)90025-6 }}$

14. F. Badalov, Method of series in nonlinear hereditary theory of viscoelasticity (Fan, Uzbekistan, 1980)

15. F. Badalov, Methods of solution of integral and integro-differential equations of hereditary viscoelasticity (Mekhnat, Uzbekistan, 1987)

16. F. Badalov and S. Ganikhanov, Vibration of hereditary-deformable elements of structure of flying vehicles (TSAI, Uzbekistan, 2000)

17. H. Robert, Scanlan, and R. Rosenbaum, Introduction to the study of Aircraft Vibration and Flutter (Macmillan, New York, 1951)

18. M. Keldysh, Selected Works. The Mechanics (Science, Moscow, 1985)

19. R. L. Bisplinghoff, H. Ashley, and R. Halfman, Aeroelasticity (Dover, New York, 1996)

20. B. Usmonov, Numerical Solution of Hereditary Equations with a Weakly Singular Kernel for Vibration Analysis of Viscoelastic Systems (2015) Proceedings of the Latvian Academy of Sciences, Section B: Natural, Exact, and Applied Sciences, 69 (6), pp. 326330. https://doi.org/10.1515/prolas-2015-0048

21. B. Usmonov, Q.Rakhimov, and A.Akhmedov, Analysis of numerical solutions of a hereditary deformable system, International Journal of Mechanical and Production Engineering Research and Development 8, 403-408 (2018)

https://doi.org/10.24247/ijmperdaug201842 